\title{
The probability of simple versus complex causal models in causal analyses
}

\author{
David Trafimow ${ }^{1}$
}

Published online: 8 April 2016

(C) Psychonomic Society, Inc. 2016

\begin{abstract}
Complex causal models, accompanied by causal analyses based on large correlation matrices, are more common in the social sciences than are simple causal models accompanied by a single correlation coefficient. The increased complexity of the former, relative to the latter, seems to carry with it an augmented scientific respectability or credibility. In contrast, the axioms of probability suggest an argument in the opposite direction.
\end{abstract}

Keywords Causal structure $\cdot$ Simple causal model $\cdot$ Complex causal model $\cdot$ Conjunction fallacy $\cdot$ Causal analysis

The social sciences are replete with examples of causal claims based on complex analyses such as mediation analysis, moderated mediation analysis, and others. In contrast, it is rare to find a causal claim based on a single correlation coefficient. Clearly, journal reviewers and editors are willing to publish causal claims in the context of complex analyses, but not to publish causal claims in the context of simple analyses. Not surprisingly, the strong trend in the social sciences is for researchers to propose and publish causal models of increasing complexity. The aim of this article is to explore whether this state of affairs is justifiable.

David Trafimow

dtrafimo@nmsu.edu

1 Department of Psychology, New Mexico State University, Las Cruces, New Mexico

\section{Overview of the issue}

It is difficult to make causal arguments on the basis of single correlation coefficients, because three types of causation could be occurring: One variable might cause the other, the other might cause the first, or some outside variable or set of variables might cause both of them. Stated more generally, the problem is that a single correlation coefficient is consistent with more than one causal structure. This problem-sometimes termed statistical indistinguishability — plagues all nonexperimental designs. As Spirtes, Glymour, and Scheines (2000) stated, "Without experimental manipulations, the resolving power of any possible method for inferring causal structure from statistical relationships is limited by statistical indistinguishability. If two causal structures can equally account for the same statistics, then no statistics can distinguish them" (p. 59).

The problem is well known, and the reviewers and editors of respectable social science journals are unlikely to let pass causal claims based on single correlations. Consequently, researchers tend not to depend on single correlations, but rather to depend on more complex mediation analyses, moderated mediation analyses, and so on. The underlying but tacit assumption is that complex statistical analyses provide superior evidence for the hypothesized causal model, as compared to a single correlation coefficient. In short, whereas a single correlation coefficient is not sufficiently convincing of a hypothesized causal model, a complex causal analysis is.

A potential problem with this claim is that researchers have shown that multiple causal structures are consistent even with complex causal analyses (e.g., Fiedler, Schott, \& Meiser, 2011; Grice, Cohn, Ramsey, \& Chaney, 2015; Kline, 2015; MacKinnon, Krull, \& Lockwood, 2000; Tate, 2015; Thoemmes, 2015; Trafimow, 2015b). Thus, increasing the size of the underlying correlation matrix and the complexity 
of the complex causal analysis does not solve the statistical indistinguishability problem. But perhaps a justification can be made using a relativity argument. That is, even if increasing the complexity of the statistical analysis does not solve the statistical indistinguishability problem, perhaps the problem is at least mitigated. After all, it is more difficult to pose alternative explanations to a complex than to a simple causal analysis. Perhaps increasing the complexity of the causal analysis also increases the probability that the causal model being tested is actually true.

\section{Correlations and probability}

Consider a single correlation coefficient that a researcher obtains. Because multiple causal mechanisms are consistent with this single correlation coefficient, the researcher's hypothesized causal model might be the reason for the finding, but it also might not be. Suppose that the hypothesized causal model is that $A$ causes $B(A \rightarrow B)$. Now suppose that another researcher hypothesizes a more complex causal model: $A$ causes $B$ causes $C$. Put in the language of mediation, the effect of $A$ on $C$ is mediated completely by $B$. To test this hypothesized causal model, the researcher obtains three correlations: $r_{A B}$, $r_{B C}$, and $r_{A C}$. For the three correlations to provide strong support for the hypothesized causal model, the following are minimal requirements.

The correlation $r_{A B}$ should be due to the causal effect of $A$ on $B$.

The correlation $r_{B C}$ should be due to the causal effect of $B$ on $C$.

The correlation $r_{A C}$ should not be due to a direct causal effect of $A$ on $C$ that is not mediated by $B$.

\section{Assume independence}

Let us commence with the assumption that the probability that $r_{A B}$ is due to the causal effect of $A$ on $B$, that the probability that $r_{B C}$ is due the causal effect of $B$ on $C$, and that the probability that $r_{A C}$ is due to the causal effect of $A$ on $C$ all are independent of each other. In addition, let us denote the probability that $A$ causes $B$ as $\pi_{1}$ and the probability that $B$ causes $C$ as $\pi_{2}$. Well, then, under the independence assumption, the probability that both propositions are true-that $A$ causes $B$ and $B$ causes $C$ - is the product of two probabilities $\left(\pi_{1} \pi_{2}\right)$. Obviously, $\pi_{1}>\pi_{1} \pi_{2}$, so we see that the probability of the more complex causal model, based on two rather than on one correlation coefficient, is decreased relative to that of the simple causal model. Even in the event that we actually have a statistically significant value for $r_{A B}$ and a statistically significant value for $r_{B C}$, it is more likely that one causal pathway is true (e.g., $A \rightarrow B$ ) than that two causal pathways are true $(A \rightarrow B$ and $B \rightarrow C)$.

Matters continue to worsen as we consider $r_{A C}$. Suppose that we denote the probability that this correlation is due to a direct causal effect of $A$ on $C$ as $\pi_{3}$. Thus, under the independence assumption, $\pi_{1}, \pi_{2}$, and $\pi_{3}$ all are independent of each other.

To fit the complex causal model, the researcher desires for $r_{A C}$ not to be directly causal, and so the probability is $1-\pi_{3}$. To make the complete causal model work, all three correlations have to have the appropriate causal status, so the probability is $\pi_{1} \pi_{2}\left(1-\pi_{3}\right)$. We can set up an inequality: $\pi_{1}>\pi_{1} \pi_{2}>\pi_{1} \pi_{2}\left(1-\pi_{3}\right)$. Clearly, as the causal model increases in complexity, an increasing number of correlation coefficients have to be due to the "right" reasons and not to the "wrong" reasons - these correlation coefficients have to have the appropriate causal status - and the probability that the complex causal model is true decreases accordingly.

To see the strength of the combined effect, let us insert some numbers. Ideally, to support the causal model, we would like $\pi_{1}$ and $\pi_{2}$ to be large numbers and $\pi_{3}$ to be a small number. So let us assign numbers of $.75, .75$, and .25 , respectively. We arrive at the following solution: $\pi_{1} \pi_{2}\left(1-\pi_{3}\right)=.75 .75(1$ $-.25)=.42$. As compared to the probability of .75 for the simple $A \rightarrow B$ model, the probability of .42 for the complete causal model is unfavorable. Arguably, the probability of .42 for the complete causal model is unfavorable in an absolute sense, even without regard to the simple $A \rightarrow B$ model. And of course, matters worsen considerably with less favorable (but perhaps more reasonable) numbers. For example, if we assume $.60, .60$, and .40 , respectively, the final result is $.60 .60(1-.40)=.22$.

To illustrate more generally the extent to which a simple causal model that depends on one correlation is more likely to be true than a complex causal model that depends on three correlations, it is useful to peruse Fig. 1, where $\pi_{1}$ and $\pi_{2}$ are set equal to each other and allowed to vary from .1 to 1.0, along the horizontal axis. In addition, $\pi_{3}$ is set at $.25, .50$, or .75. The vertical axis represents the probability that a single correlation is causal $\left(\pi_{1}\right)$ minus the probability that a more complex set of correlations represents the true state of causation $\left[\pi_{1} \pi_{2}\left(1-\pi_{3}\right)\right]$. In other words, the vertical axis represents the probability difference between the simple and complex models, or $\pi_{1}-\pi_{1} \pi_{2}\left(1-\pi_{3}\right)$. It is easy to see that under all conditions (except when $\pi_{1}$ equals zero, or $\pi_{2}$ equals 1 and $\pi_{3}$ equals 0 , which is not included in Fig. 1 ), the simple causal model is more likely to be true than the complex causal model, consistent with the inequality described earlier $\left[\pi_{1}>\pi_{1} \pi_{2}>\pi_{1} \pi_{2}\left(1-\pi_{3}\right)\right]$.

Figure 1 shows that the probability difference is reduced when $\pi_{1}$ and $\pi_{2}$ are small, and this fact could be argued to support that perhaps complex causal models are not much less 


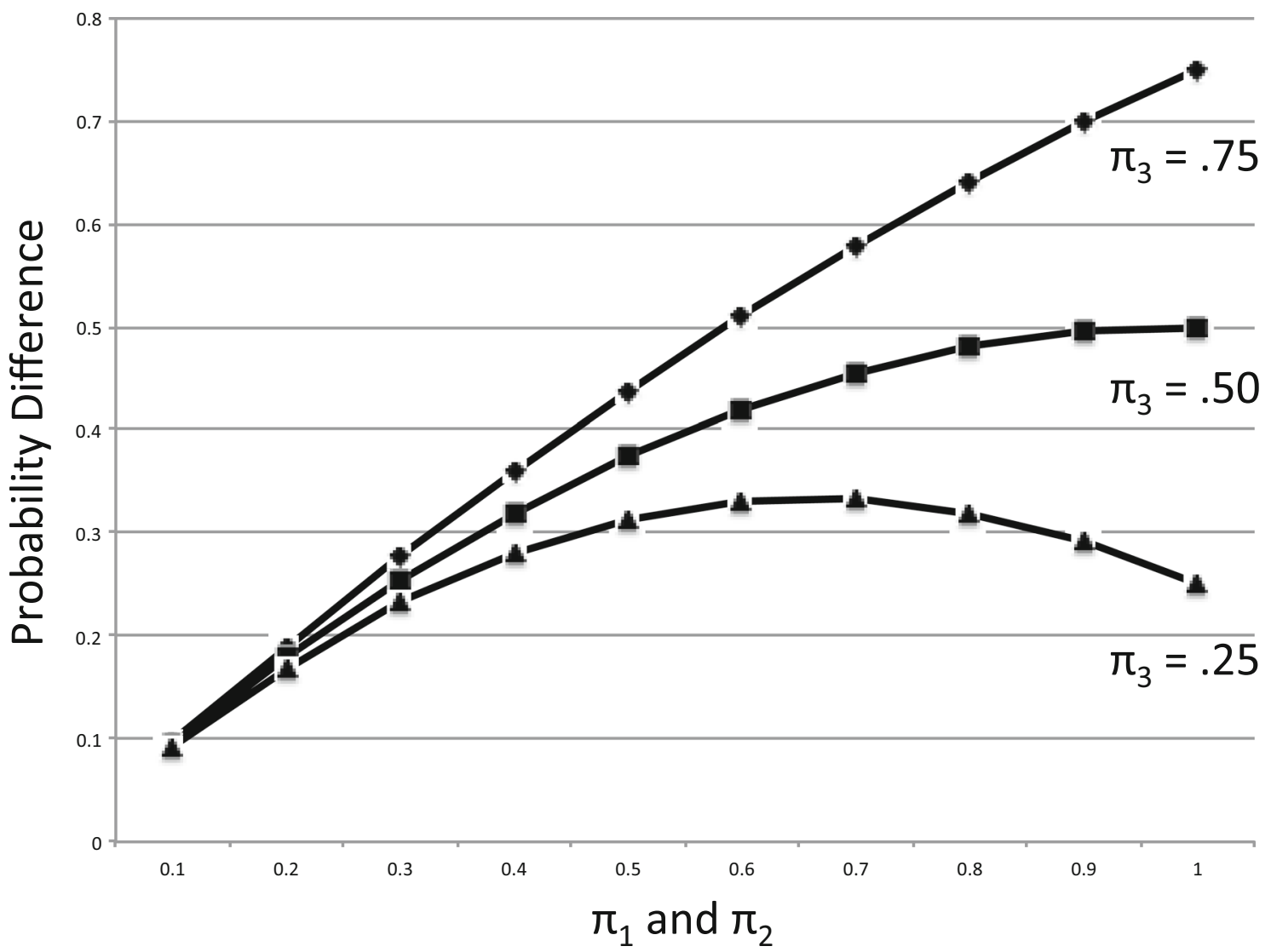

Fig. 1 In this figure, $\pi_{1}$ and $\pi_{2}$ are set equal to each other and allowed to vary from .1 to 1.0 along the horizontal axis. In contrast, $\pi_{3}$ is set at .25 , .50 , or .75 . The probability difference, which is $\pi_{1}-\pi_{1} \pi_{2}\left(1-\pi_{3}\right)$, varies

likely to be true than simple causal models under this condition. The problem here is that when $\pi_{1}$ and $\pi_{2}$ are small, neither a simple nor a complex causal model is likely to be true.

An additional factor that decreases the probability difference, at least relative to the top curve in Fig. 1, is when $\pi_{3}$ takes on a small rather than a large value. However, when $\pi_{1}$ and $\pi_{2}$ are intermediate, the probability difference nevertheless remains substantial. Therefore, for the complex causal model both to have a good chance of being true and not to be at a substantial disadvantage relative to the simple causal model, the researcher really does need to have a combination of very large values for $\pi_{1}$ and $\pi_{2}$ and a very small value for $\pi_{3}$.

The foregoing reasoning can be continued to apply to causal models of any complexity whatsoever. Suppose that there are four variables $(A, B, C$, and $D)$, so that the relevant correlation matrix contains the following six correlations: $r_{A B}, r_{A C}$, $r_{A D}, r_{B C}, r_{B D}$, and $r_{C D}$. Even if we posit ridiculously favorable numbers for $\pi_{1}$ through $\pi_{6}$, the probabilities work out unfavorably. Suppose, for example, we assign probabilities of 9 for all of the correlations that are supposed to be due to direct causation, and probabilities of .1 for all correlations that are not supposed to be due to direct causation. Nevertheless, the final probability for the complete causal model turns out to be along the vertical axis as a function of where $\pi_{1}$ and $\pi_{2}$ are set and where $\pi_{3}$ is set

.53, which is not impressive. Obviously, if more reasonable probabilities are assigned, the final probability decreases even further. For example, if we use .75 for the correlations that are supposed to be due to direct causation and .25 for the correlations that are not (which still seems optimistic), the final value is .18.

\section{Assume dependence}

Thus far, I have assumed that the probability that any correlation is causal is independent of the probability that any other correlation in the model is causal. Let us make up a trivial causal model with dependence focused on social science researchers: Researchers publishing in prestigious journals causes jealousy on the part of colleagues, which, in turn, causes them to make more negative comments about the researchers. In addition, suppose that an investigatory team tests this model using a nonexperimental paradigm and obtains correlations between all of the variables. In this example, let us designate the correlation between publishing in prestigious journals and colleague jealousy as $r_{A B}$, the correlation between colleague jealousy and negative comments as $r_{B C}$, and the 
direct correlation between publishing in prestigious journals and negative comments as $r_{A C}$. Is there reason to believe that any one of these correlations is more or less likely to be causal if one of the other ones is causal? For example, suppose that publishing in prestigious journals really does cause colleague jealousy and that colleague jealousy really does cause more negative comments. Should this influence the probability that the correlation between publishing and negative comments is due to a direct causal influence of publishing on negative comments? Although I do not see any reason to assume this, let us play the game anyhow.

Consistent with the nomenclature used earlier, let us designate $\pi_{1}$ as the probability that $r_{A B}$ is causal, $\pi_{2}$ as the probability that $r_{B C}$ is causal, and $\pi_{3}$ as the probability that $r_{A C}$ is directly causal (not mediated by $B$ ). Suppose that $\pi_{1}$ and $\pi_{2}$ are independent but that $\pi_{3}$ increases if the other two correlations really are causal. We considered earlier an example in which $\pi_{1}, \pi_{2}$, and $\pi_{3}$ were $.75, .75$, and .25 , respectively, so that the probability of the complete causal model being correct was .42 , under the assumption of complete independence. Now, however, suppose that the probability that the correlation between publishing in prestigious journals and negative comments is causal $\left(r_{A C}\right)$ is conditional on the other two correlations, so that the conditional $\pi_{3}$ is .75 . In that case, the probability that the complete causal model is correct reduces from the .42 we saw earlier, to an even lower value of .14 .

It is possible for dependence to be favorable as well as unfavorable for the complex causal model, though with a proviso. Recall that we used quite favorable values for $\pi_{1}, \pi_{2}$, and $\pi_{3}$ to put the complex causal model in a good light. Regarding $\pi_{3}$, since it is favorable for the complex causal model if this is a low number, we used .25. Suppose that dependence works to decrease this value still further, so that the conditional value is .10 instead of .25 . How much does this extremely favorable assumption increase the probability that the complete causal model is correct? The new value is .51 , which is a small increase over the value of .42 that we obtained under the assumption of complete independence.

Suppose there are four variables in the causal model, so that six correlations have to go the right way with respect to their causal status; that there are five variables in the causal model, so that there are ten correlations; or that there are six variables, leading to 15 correlations. Under the complete-independence assumption, and assuming large probabilities of causation of .75 where large values favor the causal model, and small probabilities of causation of .25 where small values favor the model, the probability of the complete causal model being correct nevertheless decreases as the number of variables increases. Thus, when there are four variables, the probability of the complete causal model being correct equals .18 (as we saw earlier); when there are five variables, the probability decreases to .056; and when there are six variables, the probability decreases to .013. Possibly, these numbers could increase somewhat assuming just the right sort of dependence, as in the foregoing example. But we also saw an example in which dependence reduced the overall support for the causal model, and in a substantial way, too. More generally, if we make generous assumptions about the values of the probabilities involving the causal status of the correlations, there is more room for dependence to detract from support for the model than for it to support the model. If we make less generous assumptions about the values of the probabilities involving the causal status of the correlations, on the one hand, this trade-off becomes less unfavorable for the causal model, but on the other hand, less generous assumptions become a problem for the causal model in their own right.

The main message echoes the one that we perceived under the assumption of independence, though with complications: Specifically, the probability that causal models are true decreases as they become more complex, and the decrease becomes extreme quickly as more variables are added to the model.

\section{Discussion}

The remainder of the present article considers four issues. First, on the basis of discussions I have had, I included a subsection with potential counterarguments and my responses to them. Second, I consider why complex causal models seem more likely to be true than simple ones, despite the strongly contradictory nature of the foregoing analysis. Third, in all of the cases considered above, the complex models subsumed the simpler ones, but this need not be so, in which case epistemological factors can qualify the conclusions reached earlier. Fourth, I place the foregoing argument in the context of a Bayesian perspective that considers both a priori and a posteriori probability.

Counterarguments One might claim that when causal models are derived from a more general theory, they are buttressed by the theory as well as by the data, thereby lending credence to even complex causal models. In the case of causal models that actually are derived from a more general theory that has received independent empirical support, this counterargument has some validity; data of almost any kind are strengthened by strong theory. However, in the vast majority of published causal models, this condition is not met, so the causal model is the theory. Perforce, a circularity issue arises. The theory is supposed to support the validity of the causal analysis, and the causal analysis is supposed to support that the theory is true.

An additional counterargument stems from the claim that researchers who test complex causal models are not really proposing "causation." This claim seems disingenuous. The use of words such as "mediate" and others, as well as the fact 
that arrows are used in the diagrams, all support the proposition that the claims made really are causal. In addition, the Discussion sections of such articles invariably make recommendations that, at least at the tacit level, assume causation. The researchers might recommend an intervention based on a variable they have identified, but why recommend an intervention based on an identified variable in the absence of conviction that the variable is causal? In addition, it is easy to find researchers who have made outright causation claims based on such analyses (e.g., Cohen, Cohen, West, \& Aiken, 2003; Hoza et al., 2013; McClendon, 1994; Rogers-Sirin, 2013).

It is possible to argue that the value of complex causal analysis is entirely different than what is usually assumed. For example, Fiedler, Schott, and Meiser (2011) are blunt in stating that the value is not in supporting causation, but rather that if one starts with the assumption that the causal model is true, it is possible to assign effect sizes to the various arrows in the diagram. Note the shift here. No longer is the causal model a hypothesis to be tested: It is an assumed fact! Even in this fait accompli case, my issue of multiple correlations remains. That is, in a simple $A \rightarrow B$ causal model, there is only a single correlation coefficient, and the measurement error might not be too great (especially with correction for attenuation due to unreliability). In contrast, as the causal model becomes more complex, the calculated effect sizes for each causal pathway in the diagram depend on multiple correlation coefficients. Thus, what are hopefully small errors multiply, and the calculated effect sizes become increasingly less likely to represent reality, even under the fait accompli assumption and even with correction for attenuation due to unreliability (see Trafimow, Rice, \& MacDonald, 2011, for mathematical analyses and computer simulations).

\section{Reasons for giving credence to complicated causal struc-} tures The foregoing analysis indicates that a simple causal model based on a single correlation coefficient is more likely to be true than a complex causal model based on several correlation coefficients. And yet it is extremely unlikely that a researcher could publish a simple causal model accompanied by a single correlation coefficient, whereas respectable social science journals are replete with complex causal models accompanied by complex path diagrams based on large correlation matrices. Given that a simple causal model is much more likely to be true than is a complex one, why do journal editors and reviewers favor complexity?

There are several possible answers. The interaction between the cognitive limitations of social science researchers and their training provides one possible answer. Researchers are trained to look for alternative explanations and also to memorize rules. In the case of a single correlation coefficient, it is easy to find outside variables that might correlate with both measured variables, thereby providing fodder for alternative explanations. It also is easy to apply the rule from introductory psychology: "Correlation does not mean causation!" When faced with a complex causal model supported by a complex statistical analysis, it is more difficult to figure out alternative explanations, because there are more correlations to explain. Also, no rule from introductory psychology, or even from graduate school, states: "A path diagram does not mean causation!" In fact, much graduate training supports the idea that a path diagram means causation. Whether through the ease of generating alternative explanations or the ease of applying a rule, social scientists' cognitive limitations suggest that a complex causal model accompanied by a complex statistical analysis based on a large correlation matrix will be looked upon more favorably than a simple causal model (e.g., $A \rightarrow B$ ) accompanied by a single correlation coefficient.

A second, but related, reason is the "only-game-in-town fallacy." Philosophers often mention this fallacy in the context of arguments about God (Sober, 2001; Trafimow, 2015a). A typical argument involves pointing out something that science cannot explain and saying, "See, God is the only explanation!" The fallacy is that a person's not thinking of an alternative explanation does not validly force belief in the particular explanation at hand. In the context of complex causal models, it might be that some journal editors and reviewers resort to the only-game-in-town fallacy as a way to handle the cognitive complexity of attempting to explain multiple correlation coefficients.

A third reason is the "conjunction fallacy," whereby multiple characteristics that people perceive as being representative are deemed to be more likely than a single characteristic (Tversky \& Kahneman, 1983). To quote a friend's satirical example, people think that the probability that a man is a doughnut-dunking policeman is greater than the probability that a man is a policeman, because "doughnut-dunking policeman" seems particularly representative. Successful purveyors of complex causal models tend to be good writers who are able to make the various variables seem representative of an overall theme, thereby increasing the probability that the conjunction fallacy will be committed. But as we have seen, as more correlations need to be due to the "right" reasons, the probability that the causal model is correct decreases.

Epistemology It is possible to interpret my argument as favoring all simple models over all complex models, but this would be going too far. As an example, consider the correlation between ice cream sales and murder. ${ }^{1}$ It might be tempting to posit that ice cream sales cause murder or that murder causes people to buy ice cream. Alternatively, we might consider a more complex argument that temperature influences both ice cream sales and murder. This would seem to be a clear case in which the more complex model has more

\footnotetext{
${ }^{1}$ I thank an anonymous reviewer for this example, as well as for suggesting this discussion.
} 


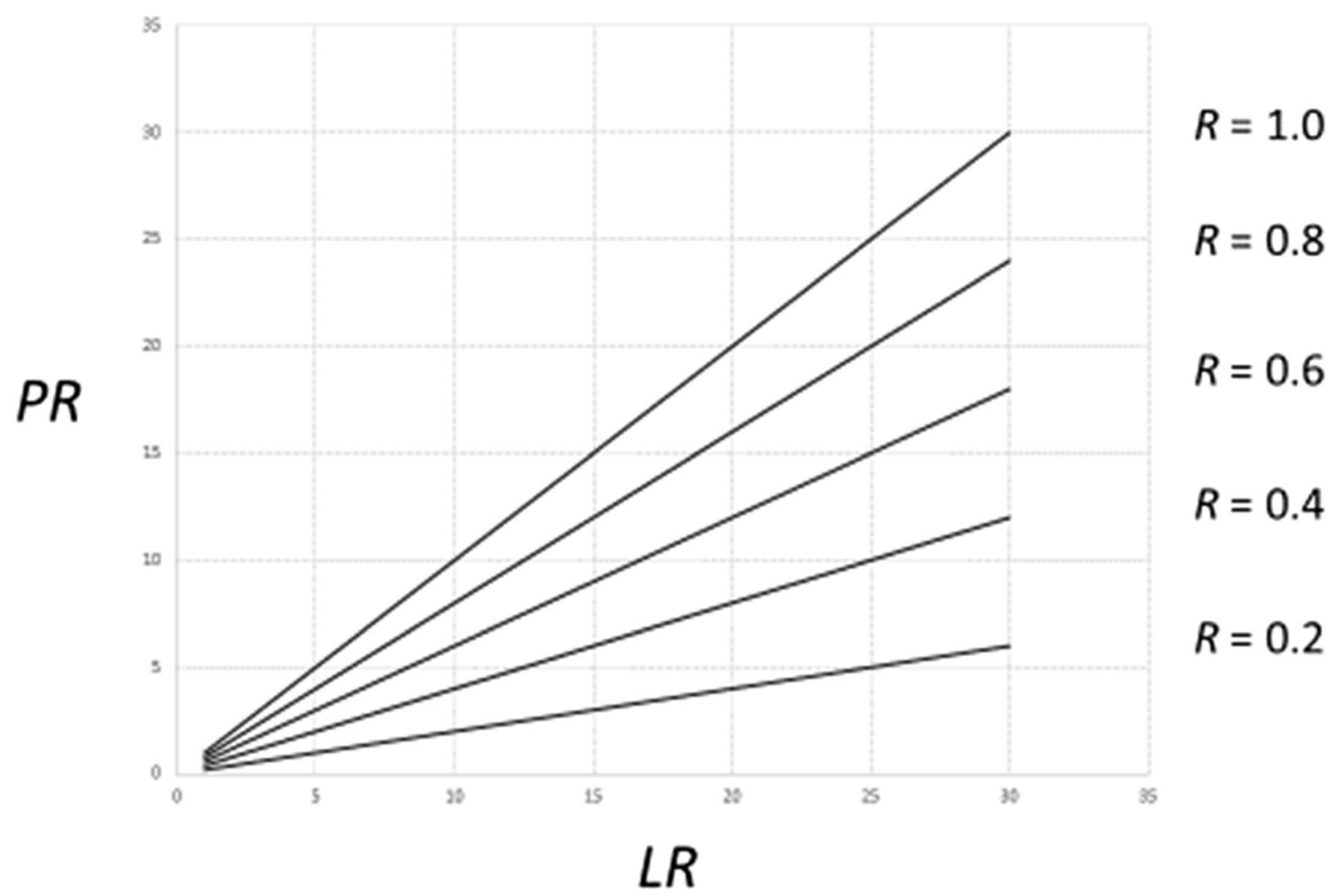

Fig. 2 The a posteriori ratio $(P R)$ as a function of the a priori ratio $(R)$ and the likelihood ratio $(L R)$

verisimilitude than does either simple model. Sometimes, the epistemological case for a complex model overwhelms the probabilistic issue.

But one issue needs to be considered to put the foregoing epistemological example in its proper context: Specifically, the more complex model in the example does not subsume either of the simple models. If it did, the more complex model would be less probable than a simple model that it subsumes, even taking epistemological factors into consideration. For example, if the model had been that high temperatures cause ice cream sales to increase, which in turn causes murders to increase, or if the model had been that high temperatures cause murders to increase, which in turn causes ice cream sales to increase, either of these actually would be less likely to be true than the simple models.

In general, the probability of a complex model will be less than that of a simple model in those cases in which the complex model subsumes the simple model, as we have assumed throughout. Also, in cases of equal epistemological status for the simple and complex models, the simple models will be more likely to be true than the complex models, even if the simple model is not subsumed by the complex one. But in cases in which the epistemological statuses of the two models are unequal, and in which the complex model does not subsume the simpler one, it is possible for epistemological factors to increase the relative plausibility of complex models. Nevertheless, even taking this into account, there are two potential problems with such use of epistemological considerations. First, if a researcher wishes to use epistemology to support complex models, the epistemological argument needs to become increasingly convincing to overcome the probabilistic disadvantage of complex models as their complexity increases, a matter to be explored in more detail in the subsequent subsection. Second, if the epistemological considerations are too convincing a priori, the researcher risks running into the problem that none of the causal pathways he or she posits actually is interesting. After all, when a model includes many hypothesized causal pathways, all of them need to be likely to be true for the conjunction of all of them to have a reasonable likelihood of being true. Nevertheless, if the data collected are of such high quality so as to provide a convincing case for a model that otherwise would not be considered convincing, that can be a major triumph for the researcher (Trafimow, 2003). The issue of a priori versus a posteriori probabilities deserves its own subsection, which is presented next.

A Bayesian context ${ }^{2}$ An epistemological factor of importance in psychology is the evidence obtained in a study. From this perspective, one might consider the probabilistic implications of complexity as an a priori factor that can be strongly mitigated or even overcome by evidence. To see that this is possible, consider astronomy or physics theories of low a priori probability that nevertheless instill much confidence in us

\footnotetext{
${ }^{2}$ I thank Klaus Fiedler for suggesting this discussion.
} 
today, because of the overwhelming supporting evidence we now have. Thus, although I wish for researchers to be skeptical about complex models, the purpose of the present subsection is to show how to avoid too much skepticism.

The famous theorem by Bayes provides a simple way to jointly consider the a priori probability of a model and the evidence obtained. The theorem takes various forms, and it is convenient to use the ratio form now, presented as Eq. 1. In that equation, the idea is to obtain the a posteriori ratio $(P R)$, which is the probability that the model is true, given the evidence $[P(M \mid E)]$, divided by the probability that the model is not true, given the evidence $[P(\sim M \mid E)]$. This ratio is the product of two other ratios. First, there is the a priori ratio $(R)$, which is the quotient of the a priori probability that the model is true $[P(M)]$ divided by the a priori probability that the model is not true $[P(\sim M)]$. Second, there is the likelihood ratio $(L R)$, which is the probability of the evidence given that the model is true $[P(E \mid M)]$ divided by the probability of the evidence given that the model is not true $[P(E \mid \sim M)]$.

$$
\frac{P(M \mid E)}{P(\sim M \mid E)}=\frac{P(M)}{P(\sim M)} \frac{P(E \mid M)}{P\left(\left.E\right|^{\sim} M\right)} \text { or } P R=(R)(L R)
$$

Given Eq. 1, consider again the example presented earlier of a complex model with three pathways, where the a priori probability of the whole complex model being true is .22, so that the probability of it not being true is $1-.22=.78$. In that case, the a priori ratio is $\frac{.22}{.78}=.28$. What is the quality of evidence needed to provide a strong a posteriori case, despite the poor a priori beginning? Suppose that the researcher has obtained evidence of high quality; that is, the probability of the evidence is much greater if the model is true than if it is not true (Trafimow, 2003). For example, suppose that the evidence is threefold more likely if the model is true than if it is not true $(L R=3)$. In that case, $P R$ would be the product of .28 and 3 , which equals .84. In other words, even with reasonably strong evidence, the a priori ratio is so poor that reasonably strong evidence fails to bring us even to the point of indifference (where $P R$ equals 1 ). It would take evidence that is 4.55 times as likely, given that the model is true than that it is not true, to bring us to the point of indifference. And matters get worse when we consider what it might take to have a strong case for the model. Bayesians have suggested several cutoffs for the ratio needed to strongly support a model, with 10 being a common one (e.g., Etz \& Vandekerckhove, 2016). If we use 10 , then the $L R$ needed to get there from the starting point of .28 is 35.71 . Although this may be possible, collecting data of such quality constitutes a difficult challenge for researchers to overcome.

To see how the quality of the data and the complexity of the model combine more generally to influence the $P R$, consider Fig. 2, where the five curves correspond to when the a priori ratio is $.2, .4, .6, .8$, and 1.0. On the horizontal axis, $L R$ varies from 1 to 30 (I could have included higher values, but it would make the figure difficult to see in detail). The vertical axis gives $P R$. If we commence at the top curve, we see that an $L R$ of only 10 is sufficient to obtain a $P R$ of 10 . That is the good news. The bad news is that the other curves, on which the a priori ratios are lower, require an increasingly favorable $L R$ to reach the arbitrary cutoff of 10 along the vertical axis. To take the worst-case scenario illustrated in Fig. 2, when the a priori ratio is .2, even an $L R$ of 30 will be insufficient to instill confidence in the model (the required $L R$ is 50 !). Nor is the worst-case scenario particularly unlikely. Many models in psychology are much more complex than having three, or even four, variables, so assigning an a priori probability of .2 might be quite optimistic. Whether, or how often, psychology studies meet the strong epistemological requirements for overturning poor a priori beginning would be an interesting topic for future discussion.

\section{Conclusion}

In conclusion, I see two routes that are consistent with the foregoing comments. Researchers should be more open to simple causal models based on a single correlation coefficient or they should be less open to complex causal models based on many correlation coefficients. Either would be consistent with the way that probability works. The current trend of placing more credence on complex causal models based on many correlation coefficients than on simple ones based on a single correlation coefficient does not accord with the axioms of probability, and therefore is unjustifiable.

\section{References}

Cohen, J., Cohen, P., West, S. G., \& Aiken, L. (2003). Applied multiple regression/correlation analysis for the behavioral sciences (3rd ed.). Mahwah, NJ: Erlbaum.

Etz, A., \& Vandekerckhove, J. (2016). A Bayesian perspective on the reproducibility project: Psychology. PLOS ONE, 11, e149794. doi: 10.1371/journal.pone.0149794

Fiedler, K., Schott, M., \& Meiser, T. (2011). What mediation analysis can (not) do. Journal of Experimental Social Psychology, 47, 12311236. doi:10.1016/j.jesp.2011.05.007

Grice, J. W., Cohn, A., Ramsey, R. R., \& Chaney, J. M. (2015). On muddled reasoning and mediation modeling. Basic and Applied Social Psychology, 37, 214-225. doi:10.1080/01973533.2015. 1049350

Hoza, B., McQuade, J. D., Murray-Close, D., Shoulberg, E., Molina, B. S. G., Arnold, L. E., \& Swanson, J. (2013). Does childhood positive self-perceptual bias mediate adolescent risky behavior in youth from the MTA study? Journal of Consulting and Clinical Psychology, 81, 846-858. doi:10.1037/a0033536

Kline, R. B. (2015). The mediation myth. Basic and Applied Social Psychology, 37, 202-213. doi:10.1080/01973533.2015.1049349 
MacKinnon, D. P., Krull, J. L., \& Lockwood, C. M. (2000). Equivalence of the mediation, confounding and suppression effect. Prevention Science, 1, 173-181. doi:10.1023/A:1026595011371

McClendon, M. J. (1994). Multiple regression and causal analysis. Itasca, IL: F. E. Peacock.

Rogers-Sirin, L. (2013). Segmented assimilation and attitudes toward psychotherapy: A moderated mediation analysis. Journal of Counseling Psychology, 60, 329-339. doi:10.1037/a0032453

Sober, E. (2001). Core questions in philosophy: A text with readings (3rd ed.). Upper Saddle River, NJ: Prentice Hall.

Spirtes, P., Glymour, C., \& Scheines, R. (2000). Causation, prediction, and search. Cambridge, MA: MIT Press.

Tate, C. U. (2015). On the overuse and misuse of mediation analysis: It may be a matter of timing. Basic and Applied Social Psychology, 37, 235-246. doi:10.1080/01973533.2015.1062380

Thoemmes, F. (2015). Reversing arrows in mediation models does not distinguish plausible models. Basic and Applied Social Psychology, 37, 226-234. doi:10.1080/01973533.2015.1049351
Trafimow, D. (2003). Hypothesis testing and theory evaluation at the boundaries: Surprising insights from Bayes's theorem. Psychological Review, 110, 526-535. doi:10.1037/0033-295X.110. 3.526

Trafimow, D. (2015a). The only game in town fallacy and alternative explanations for experimental findings. American Research Journal of Humanities and Social Sciences, 1, 40-42. Retrieved from www.arjonline.org/papers/arjhss/v1-i1/6.pdf

Trafimow, D. (2015a). What if planetary scientists used mediation analysis to infer causation? Basic and Applied Social Psychology, 37, 197-201. doi:10.1080/01973533.2015.1064290

Trafimow, D., Rice, S., \& MacDonald, J. A. (2011). An investigation of the accuracy of standardized path coefficients. Journal of General Psychology, 138, 201-214. doi:10.1080/00221309.2011.584584

Tversky, A., \& Kahneman, D. (1983). Extension versus intuitive reasoning: The conjunction fallacy in probability judgment. Psychological Review, 90, 293-315. doi:10.1037/0033-295X.90.4.293 